\title{
A SAFE SUPERVISORY FLIGHT CONTROL SCHEME IN THE PRESENCE OF CONSTRAINTS AND ANOMALIES
}

\author{
GIUSEPPE FRANŻ̀ $^{a, *}$, ANGELO FURFARO $^{a}$, MASSIMILIANO MATTEI $^{b}$, \\ VALERIO SCORDAMAGLIA ${ }^{c}$ \\ ${ }^{a}$ Dipartimento di Informatica, Modellistica, Elettronica e Sistemistica \\ Università della Calabria, Via Pietro Bucci, Cubo 42-C, Rende (CS), 87036, Italy \\ e-mail: $\{$ franze, a.furfaro\} @dimes.unical.it \\ ${ }^{b}$ Ingegneria Industriale e dell'Informazione \\ Seconda Università degli Studi di Napoli, Real Casa dell'Annunziata Via Roma, 29, Aversa (CE), 81031, Italy \\ e-mail: massimiliano.mattei@unina2.it \\ ${ }^{c}$ Dipartimento di Ingegneria, delle Infrastrutture e dell'Energia Sostenibile \\ Università degli Studi di Reggio Calabria, Reggio Calabria, Via Graziella, loc. Feo di Vito, 89060, Italy \\ e-mail: valerio.scordamaglia@unirc.it
}

\begin{abstract}
In this paper the hybrid supervisory control architecture developed by Famularo et al. (2011) for constrained control systems is adopted with the aim to improve safety in aircraft operations when critical events like command saturations or unpredicted anomalies occur. The capabilities of a low-computational demanding predictive scheme for the supervision of non-linear dynamical systems subject to sudden switchings amongst operating conditions and time-varying constraints are exploited in the flight control systems framework. The strategy is based on command governor ideas and is tailored to jointly take into account time-varying set-points/constraints. Unpredictable anomalies in the nominal plant behaviour, whose models fall in the category of time-varying constraints, can also be tolerated by the control scheme. In order to show the effectiveness of the proposed approach, simulations both on a high altitude performance demonstrator unmanned aircraft with redundant control surfaces and the P92 general aviation aircraft are discussed.
\end{abstract}

Keywords: supervisory control, command governor, failures, fault tolerant control, flight control.

\section{Introduction}

When applied to complex systems traditional feedback control algorithms may result in unsatisfactory performance, or even instability in the occurrence of actuators, sensors or other system component behaviour anomalies. By referring to Isermann and Ballè (1997), the fault management area concerns the following items:

- fault: an unpermitted deviation of at least one characteristic property or parameter of the system form the acceptable/usual/standard condition,

- failure: a permanent interruption of a system ability to perform a required function under specified operating conditions,

${ }^{*}$ Corresponding author
- malfunction: an intermittent irregularity in the fulfilment of a system's desired function.

Possible fault sources are due to permanent causes (such as wear or damage of the components) or temporary causes (due to a transient change in the working conditions); see, e.g., the work of Patton (1997).

To address these issues, a fault tolerant control (FTC) scheme, whose main task is to steer/hold the plant to/into a safe and acceptable state whenever undesired events known as faults occur, needs to be built up. In view of these requirements, the design, implementation, and maintenance cost of an FTC system may be significantly higher than those pertaining to a traditional control system. Therefore, using a fault-tolerant control system is justified if safety-critical applications are dealt with (see Blanke et al., 2006). FTC is currently a complex 
research area because joint fault detection/isolation and control structure reconfiguration tasks (or a similar countermeasure) are involved. The interested reader is referred to the works of Zhang and Jiang (2008) or Steffen (2005) for detailed and up-to-date literature reviews on the methodological solutions exploited to implement FTC schemes.

The first proposed approach to the reconfiguration problem is the pseudo-inverse method (Gao and Antsaklis, 1991), applicable both to actuator and sensor faults, limited to an approximation of the nominal system matrix. Closed-loop stability could not be ensured and additional steps are necessary to fix it. In particular, model-matching methods can deal with the stabilization problem, as shown by Chen et al. (2002). This approach relies, however, on the transfer function of the system and the mathematical treatment is complex, resulting in algorithms which are difficult to put in practise. In the work of Mhaskar et al. (2008), the problem of FDI (fault detection and isolation) and FTC for nonlinear system subject to faults in the control actuators and constraints on the manipulated inputs for both the state/output-feedback cases is considered. Necessary conditions for the design of state- and output-feedback fault detection and isolation filters are derived, and reconfiguration rules are instrumental to identify the appropriate system mode accounting for the faulty actuator.

The reconfigurability system property has been treated only recently, e.g. a structural analysis based on the control energy of linear state space systems can be found in the work of Staroswiecki (2010). Of great interest is also the contribution by Seron et al. (2012), where a novel set-theoretic approach to the design of a sensor-based FTC scheme is proposed. There, the idea consists in the separation of healthy attractive invariant sets, where appropriate fault residual signal variables remain under healthy operation, from under-fault sets, where the residual variables jump when abrupt sensor faults occur in one or more groups of sensors. The computation of these sets, as well as the derivation of conditions to achieve the aforementioned separation, can be performed beforehand and depends on known system data such as plant and estimator dynamics and bounds on reference signals and disturbances. An important property of this set-based approach is that the resulting design can be guaranteed to be fault tolerant under severe sensor faults.

Finally, it is important to highlight that FTC requirements become more compelling when flight controlled systems are dealt with. In fact, considering the presence of saturating actuators, flight envelope limitations and restrictions due to comfort and safety requirements, flight control problems turn out to be subject to input and state constraints which can make the controller design a complex task (see, e.g., Magree et al., 2012).

A second important issue relies on the real-time implementation of an FTC unit. In the literature, the most popular paradigms proposed for real-time applications are based on adaptive/soft computing algorithms, extremely useful in the case of severe plant nonlinearities due to sudden switchings and fault/failures occurrences (see the work of Guo and Song (2009) and the references therein), and predictive schemes which can efficiently cope with constraints and nonlinear dynamics (Wang and Boyd, 2010; Micksh et al., 2008; Mattei et al., 2013).

Moving from the above considerations, this paper is devoted to analyze the effectiveness and drawbacks of a recently developed constrained control strategy, known as the hybrid command governor (HCG) in the flight control systems context (see the works of Famularo et al. (2011) and Franzè et al. (2013) for technical details).

The basic command governor (CG) approach is a predictive scheme where stabilization/performance is provided by the primal controller and constraint violation avoidance is separately achieved by the CG strategy (see Bemporad et al., 1997; Bemporad, 1998; Gilbert et al., 1995; Gilbert and Kolmanovsky, 1999). The $\mathrm{CG}$ is a nonlinear device that is added to a primal inner controller designed so as to exhibit stability and tracking performance in the absence of constraints. At each discrete time instant, the CG outer device computes a modified reference command that, if applied from the current instant onward, does not produce constraint violations. Such a modified reference command is computed to minimize its distance from the actual desired reference signal according to an on-line constrained optimization over a receding horizon finite time interval.

Then, a general framework capable to take care of the possible plant structure modifications that could take place during the on-line operations is considered. The proposed scheme prescribes that any change in the plant structure affects the $\mathrm{CG}$ design and, in consequence, for each plant structure variation a different $\mathrm{CG}$ unit should be in principle designed complying with the new conditions. The idea is then that a suitable supervisory unit must be designed to take care of orchestrating the switching amongst the CG candidates during the on-line operations. The real-time supervisory CG implementation capable to comply with switchings (Branicky, 1998) amongst operating conditions and time-varying constraints has been developed and its properties formally proved. In fact, a class of unpredictable plant anomalies arising in flight control can be modelled in terms of switchings and time-varying constraints.

Simulation tests are conducted both on a high altitude performance demonstrator (HAPD) unmanned aircraft nonlinear model including flexibility and the P92 small commercial aircraft. Both aircraft are subject to surface deflection limitations in terms of amplitudes and rates of 
deflections.

The paper is organized as follows. A description of the HAPD and P92 aircraft mathematical models is given in Section 2, the CG basic properties are covered in Section 3, the conditions under which CG switchings are allowed are discussed in Section 4, whereas the real-time supervisory strategy description is given in Section 5. In Section 6, simulations are described and discussed, and some conclusions end the paper.

\section{Aircraft mathematical models}

In this section, nonlinear mathematical models of a high altitude performance demonstrator (HAPD) and of a P92-S Echo Classic aircraft are detailed.

HAPD. The high altitude performance demonstrator is an over-actuated unmanned aircraft, see Fig. 1 In particular, it has three pairs of elevators divided in inboard (IB), middle (MID) and outboard (OB), two pairs of ailerons divided into inboard (IB) and outboard (OB), and two rudders, namely, the upper (SUP) and the lower (INF) rudder. Thrust is generated by eight independent electrically powered propellers.
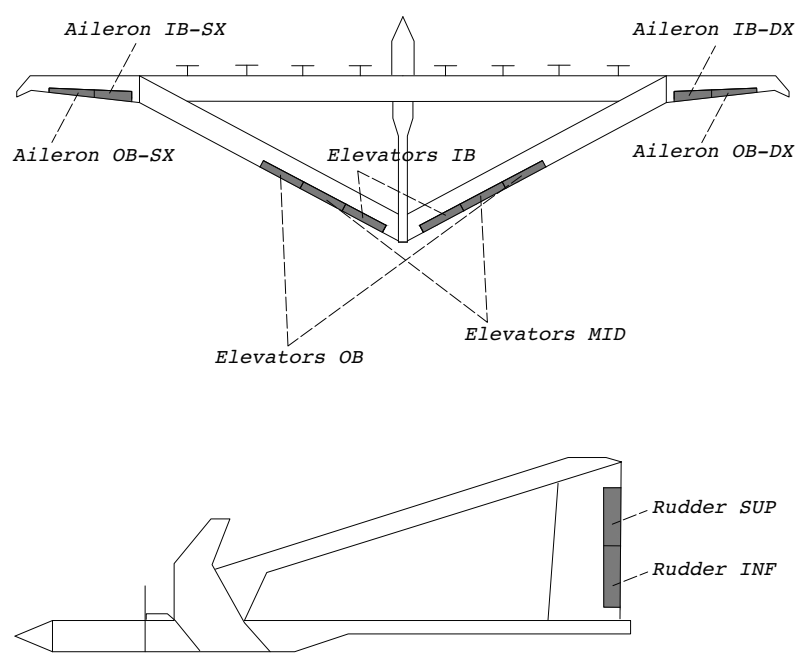

Fig. 1. HAPD model: twelve control surfaces and eight available propellers.

A mathematical model, which takes into account flexibility, was developed by the Italian Aerospace Research Center (CIRA) under the following hypotheses: the inertia matrix $I$, propellers and aerodynamic surfaces are independent from the aircraft elastic deformations; linear elastic theory can be used to model the aero-elastic dynamics; aero-elastic modes are quasi-stationary; inertial effects due to actuator forces are negligible. Under such assumptions, the polar form of the nonlinear equations of motion is (see, e.g., Stevens and Lewis, 1992)

$$
\begin{aligned}
M \dot{V}= & T \cos \alpha \cos \beta-\bar{q} S C_{D}+M g_{1}, \\
M V \dot{\beta}= & -T \cos \alpha \sin \beta+\bar{q} S C_{Y} \\
& -M V r+W g_{2}, \\
M V \cos \beta \dot{\alpha}= & -T \sin \alpha-\bar{q} S C_{L} \\
& +M V q+W g_{3}, \\
I_{x} \dot{p}-I_{x z} \dot{r}= & \bar{q} S_{b} C_{l}+q r\left(I_{y}-I_{z}\right)+p q I_{x z}, \\
I_{y} \dot{q}= & \bar{q} S_{c} C_{m}+r p\left(I_{z}-I_{x}\right) \\
& +\left(r^{2}-p^{2}\right) I_{x z}, \\
-I_{x z} \dot{p}+I_{z} \dot{r}= & \bar{q} S_{b} C_{n}+p q\left(I_{x}-I_{y}\right)-q r I_{x z}, \\
\dot{\phi}= & p+q \tan \theta \sin \phi+r \tan \theta \cos \phi, \\
\dot{\theta}= & q \cos \phi-r \sin \phi,
\end{aligned}
$$

where $\bar{q}=0.5 \rho V_{T A S}^{2}$ is the dynamic pressure, $T$ the thrust, $V_{T A S}=\left\|V_{B}-V_{W}\right\|$ the true air speed, $V_{B}=$ $\left(u_{B}, v_{B}, w_{B}\right)$ the $6 \mathrm{DoF}$ linear velocity vector, $V_{W}=$ $\left(u_{W}, v_{W}, w_{W}\right)$ the atmospheric wind velocity vector, $\omega_{B}=[p, q, r]^{T}$ the rotational velocity vector, $V=\left\|V_{B}\right\|$,

$$
\alpha=\arctan \left(\frac{w_{B}-w_{W}}{u_{B}-u_{W}}\right)
$$

the angle of attack,

$$
\beta=\arcsin \left(\frac{v_{B}-v_{W}}{V}\right)
$$

the sideslip angle, $\phi$ and $\psi$ the roll and pitch angles, $\rho$ the air density, $I_{x}, I_{y}, I_{z}, I_{x z}$ the moments and products of inertia in body axes and

$$
\begin{aligned}
g_{1}= & g(-\cos \alpha \cos \beta \sin \theta \\
& +\sin \beta \sin \phi \cos \theta+\sin \alpha \cos \beta \cos \phi \cos \theta), \\
g_{2}= & g(\cos \alpha \sin \beta \sin \theta \\
& +\cos \beta \sin \phi \cos \theta-\sin \alpha \sin \beta \cos \phi \cos \theta), \\
g_{3}= & g(\sin \alpha \sin \theta+\cos \alpha \cos \phi \cos \theta),
\end{aligned}
$$

with $g=9.8 \mathrm{~m} / \mathrm{s}^{2}$, the gravity acceleration.

Moreover, by resorting to the generalized state variables $\eta_{i}$ and $\dot{\eta}_{i}$, aero-elastic modes are modelled by means of a second order linear state space description:

$$
M_{\eta_{i}} \ddot{\eta}_{i}+\zeta_{\eta_{i}} \dot{\eta}_{i}+M_{\eta_{i}} \omega_{\eta_{i}} \eta_{i}=Q_{\eta_{i}}
$$

$i=1, \ldots, n_{a}$, where $M_{\eta_{i}}$ is the generalized mass of the $i$-th mode, $\zeta_{\eta_{i}}$ the generalized damping coefficient, $\omega_{\eta_{i}}$ the generalized natural frequency and $Q_{\eta_{i}}$ the generalized force. Notice that due to aero-elastic dynamics the aerodynamic coefficients $\left(C_{D}, C_{Y}, C_{L}, C_{l}, C_{m}, C_{n}\right)$ and generalized forces $Q_{\eta_{i}}$ depend on the $6 \mathrm{DoF}$ state variables $\left(V_{T A S}, \alpha, \beta, p, q, r\right)$, on the surfaces control deflection $\left(\delta_{\text {sup }}\right)$, and on the generalized state variables $\eta_{i}$ and 
Table 1. HPDA: main parameters.

\begin{tabular}{|c|c|c|}
\hline Parameters & Value & Units \\
\hline \hline Wing area $(\mathrm{S})$ & 13.5 & $\mathrm{~m}^{2}$ \\
\hline Wing span $\left(S_{b}\right)$ & 16.55 & $\mathrm{~m}$ \\
\hline Mean chord $\left(S_{c}\right)$ & 0.557 & $\mathrm{~m}$ \\
\hline Mass $(\mathrm{M})$ & 184.4 & $\mathrm{~kg}$ \\
\hline Elevators Slew Rates & \pm 200 & $\mathrm{deg} / \mathrm{s}$ \\
\hline Ailerons slew rates & \pm 200 & $\mathrm{deg} / \mathrm{s}$ \\
\hline Rudders slew rates & \pm 200 & $\mathrm{deg} / \mathrm{s}$ \\
\hline Ailerons deflections & \pm 25 & $\mathrm{deg}$ \\
\hline Elevators deflections & \pm 25 & $\mathrm{deg}$ \\
\hline Rudders deflections & \pm 25 & $\mathrm{deg}$ \\
\hline
\end{tabular}

$\dot{\eta}_{i}$. Finally, the thrust $T$ is assumed to be a known function of the throttle command $\delta_{T}$. For more details on the proposed HAPD mathematical model, the interested reader can refer to Scordamaglia et al. (2012).

P92-S Echo Classic. The rigid aircraft mathematical model of P92 is given by Eqns. (1)-(7) whose parameter numerical values are reported in Table 2

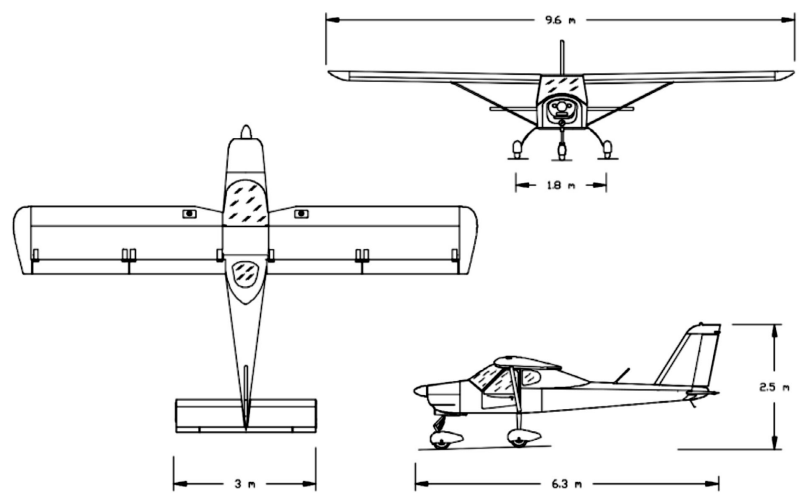

Fig. 2. Tecnam P92-S Echo Classic light aircarft.

It is important to underline that there are several sources of uncertainties in the above aircraft mathematical model: inertial parameters due to varying payloads, fuel consumption, passengers aerodynamic parameters. However, an aerodynamic aircraft characterization can be always obtained by means of wind tunnel tests or $\mathrm{CFd}$ (computational fluid dynamics) that in any case are affected by model errors.

\section{Command governor design}

Consider the following linear system:

$$
\left\{\begin{array}{l}
x(t+1)=\Phi x(t)+G g(t)+G_{d} d(t), \\
y(t)=H_{y} x(t) \\
c(t)=H_{c} x(t)+L g(t)+L_{d} d(t),
\end{array}\right.
$$

Table 2. P92-S Echo Classic: main parameters.

\begin{tabular}{|c|c|c|}
\hline Parameters & Value & Units \\
\hline \hline Wing Area $(\mathrm{S})$ & 13.2 & $\mathrm{~m}^{2}$ \\
\hline Wing span $\left(S_{b}\right)$ & 9.6 & $\mathrm{~m}$ \\
\hline Mean chord $\left(S_{c}\right)$ & 1.4 & $\mathrm{~m}$ \\
\hline Mass $(\mathrm{M})$ & 450 & $\mathrm{~kg}$ \\
\hline Elevator slew rates & \pm 80 & $\mathrm{deg} / \mathrm{s}$ \\
\hline Aileron SLEW RATEs & \pm 80 & $\mathrm{deg} / \mathrm{s}$ \\
\hline Rudder slew rates & \pm 80 & $\mathrm{deg} / \mathrm{s}$ \\
\hline Aileron deflections & \pm 30 & $\mathrm{deg}$ \\
\hline Elevator deflections & \pm 30 & $\mathrm{deg}$ \\
\hline Rudder deflections & \pm 30 & $\mathrm{deg}$ \\
\hline
\end{tabular}

where $x(t) \in \mathbb{R}^{n}$ is the state vector, $g(t) \in \mathbb{R}^{m}$ is the input vector, hereinafter called CG action, $d(t) \in \mathcal{D} \subset$ $\mathbb{R}^{n_{d}}, \forall t \in \mathbb{Z}_{+}$is the exogenous disturbance vector with $\mathcal{D}$ being a specified convex and compact set such that $0_{n_{d}} \in \mathcal{D}, y(t) \in \mathbb{R}^{m}$ is the plant output vector which is required to track $r(t), c(t) \in \mathcal{C} \subset \mathbb{R}^{n_{c}}, \forall t \in \mathbb{Z}_{+}$is the constrained output vector, with $\mathcal{C}$ a specified convex and compact set. Assume that all the eigenvalues of matrix $\Phi$ are in the open unit disk and system (11) is offset-free, i.e., $H_{y}\left(I_{n}-\Phi\right)^{-1} G=I_{m}$, with $I_{n}$ and $I_{m}$ the identity matrices of order $n$ and $m$, respectively. The CG design deals with the problem of generating, at each time instant $t$, the set-point $g(t)$ as a function of the current state $x(t)$ and reference $r(t)$ such that the constraints are always fulfilled along the system trajectories and possibly $y(t) \approx r(t)$

In view of the linearity of (11), it is possible to separate the effects of the initial conditions and input from those of disturbances so that the disturbance-free solutions of (11) to a constant command $g(t)=w$ are

$$
\begin{gathered}
\bar{x}_{w}:=\left(I_{n}-\Phi\right)^{-1} G w, \\
\bar{y}_{w}:=H_{y}\left(I_{n}-\Phi\right)^{-1} G w, \\
\bar{c}_{w}:=H_{c}\left(I_{n}-\Phi\right)^{-1} G w+L w .
\end{gathered}
$$

Consider the following set recursions:

$$
\begin{aligned}
\mathcal{C}_{0} & :=\mathcal{C} \sim L_{d} \mathcal{D}, \\
\mathcal{C}_{k} & :=\mathcal{C}_{k-1} \sim H_{c} \Phi^{k-1} G_{d} \mathcal{D}, \\
& \vdots \\
\mathcal{C}_{\infty} & :=\bigcap_{k=0}^{\infty} \mathcal{C}_{k},
\end{aligned}
$$

where $\mathcal{A} \sim \mathcal{E}$ is defined as $\{a: a+e \in \mathcal{A}, \forall e \in \mathcal{E}\}$. It can be shown that the sets $\mathcal{C}_{k}$ are nonconservative restrictions of $\mathcal{C}$ such that $\bar{c}(t) \in \mathcal{C}_{\infty}, \forall t \in \mathbb{Z}_{+}$, implies that $c(t) \in \mathcal{C}, \forall t \in \mathbb{Z}_{+}$. Thus, one can consider only disturbance-free evolutions of the system and adopt a 
"worst-case" approach. By introducing the following sets:

$$
\mathcal{C}^{\delta}:=\mathcal{C}_{\infty} \sim \mathcal{B}_{\delta}, \quad \mathcal{W}^{\delta}:=\left\{w \in \mathbb{R}^{m}: \bar{c}_{w} \in \mathcal{C}^{\delta}\right\}
$$

where $\mathcal{B}_{\delta}$ is a ball of radius $\delta$ centered at the origin, we shall assume that there exists a vanishing $\delta>0$ such that $\mathcal{W}^{\delta}$ is nonempty. In particular, $\mathcal{W}^{\delta}$ is the closed and convex set of all commands whose corresponding steady-state solutions satisfy the constraints with a tolerance margin $\delta$.

The CG algorithm provides at each time step a constant virtual command $g(\cdot) \equiv w$, with $w \in \mathcal{W}^{\delta}$, such that the corresponding disturbance-free evolution fulfils the constraints over a semi-infinite horizon and its "distance" from the constant reference is minimal. In this respect consider the set

$$
\mathcal{V}(x)=\left\{w \in \mathcal{W}^{\delta}: \bar{c}(k, x, w) \in \mathcal{C}_{k}, \forall k \in \mathbb{Z}_{+}\right\},
$$

where

$$
\bar{c}(k, x, w)=H_{c}\left(\Phi^{k} x+\sum_{i=0}^{k-1} \Phi^{k-i-1} G w\right)+L w
$$

is the constrained output vector at time $k$ from the initial condition $x$ under the constant command $g(\cdot) \equiv w$. The CG output is chosen according to the solution of the following constrained optimization problem:

$$
g(t)=\arg \min _{w \in \mathcal{V}(x(t))}\|w-r(t)\|_{\Psi}
$$

with $\|w\|_{\Psi}:=w^{T} \Psi w, \Psi=\Psi^{T}>0$ being a suitable weighting matrix. Theoretical studies along these lines on CGs appeared in the works of Gilbert et al. (1995), Gilbert and Kolmanovsky (1999), Bemporad (1998), Angeli and Mosca (1999), Angeli et al. (2001) and Garone et al. (2010).

\section{Hybrid command governors}

In this section a supervisory based CG framework capable to deal with the plant structure modifications (references and constraint configurations) that could take place during the on-line operations is introduced. The basic CG scheme is generalized to both time-varying set-points and time-varying constraint paradigms, in such way that the properties of the basic CG are preserved. To this end, a suitable supervisory unit is designed for orchestrating the switching amongst the CG candidates during the on-line operations. The overall technique is termed a hybrid CG (HCG) control scheme.

Consider the discrete-time nonlinear system model

$$
x_{p}(t+1)=f\left(x_{p}(t), u(t)\right),
$$

where $x_{p}(t) \in X \subseteq \mathbb{R}^{n}$ and $u(t) \in U \subseteq \mathbb{R}^{m}$ are the system state and control vectors, respectively, $X, U$ being convex and compact sets. Assume that $f(x, u)$ is continuously differentiable in its arguments and that the plant 13 could operate in $N$ pre-specified working regions, characterized by $N$ equilibrium points, denoted as $\left(x_{p_{i}}^{e q}, u_{i}^{e q}\right), i=1, \ldots, N$.

Suppose that for each equilibrium couple $\left(x_{p_{i}}^{e q}, u_{i}^{e q}\right)$ a linearized model from (13) can be derived (Khalil, 1996),

$$
\delta x_{p}(t+1)=A_{i} \delta x_{p}(t)+B_{i} \delta u(t)+F_{i}\left(\delta x_{p}(t), \delta u(t)\right),
$$

where

$$
A_{i}=\left.\frac{\partial f}{\partial x_{p}}\left(x_{p}, u\right)\right|_{\left(\begin{array}{c}
x_{p}=x_{p_{i}}^{e q} \\
u=u_{i}^{e q}
\end{array}\right)}
$$

and

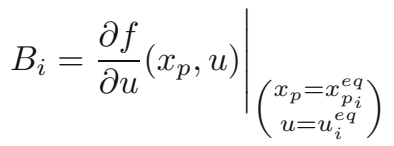

are Jacobian matrices with $\delta x_{p}=x_{p}-x_{p_{i}}^{e q}$ and $\delta u=$ $u-u_{i}^{e q}$. Then $F\left(\delta x_{p}, \delta u\right)$ is the Taylor series remainder term and by means of continuity arguments we have that

$$
\frac{\left\|F_{i}\left(\delta x_{p}, \delta u\right)\right\|_{2}}{\left\|\left[\delta x_{p}^{T}, \delta u^{T}\right]^{T}\right\|_{2}} \rightarrow 0 \text { as }\left\|\left[\delta x_{p}^{T}, \delta u^{T}\right]^{T}\right\|_{2} \rightarrow 0 .
$$

Therefore, for any $\gamma^{i}>0$ there exist $r^{i}>0$ such that

$$
\frac{\left\|F_{i}\left(\delta x_{p}, \delta u\right)\right\|_{2}}{\left\|\left[\delta x_{p}^{T}, \delta u^{T}\right]^{T}\right\|_{2}}<\gamma^{i}, \quad \forall\left\|\left[\delta x_{p}^{T}, \delta u^{T}\right]^{T}\right\|_{2}<r^{i}
$$

4.1. Time-varying set-points. Suppose that references are allowed to belong to a finite levels set (see the work of Bacconi et al. (2007) for details),

$$
r \in \mathcal{R}:=\left\{r_{1}, \ldots, r_{q}\right\}, \quad r_{i} \in \mathbb{R}^{m},
$$

$i=1, \ldots, q$, and for each $i$-th linearized model a single primal controller/reference governor unit $C G_{i}$ is derived with $\mathcal{W}_{i}^{\delta}, i \in \mathcal{N}:=\{1,2, \ldots, N\}$, being the set of all commands whose corresponding steady-state solutions satisfy the constraint with margin $\delta$.

In order to ensure that each set point inside $\mathcal{R}$ can be tracked, let us suppose that

$$
\mathcal{R} \subset \bigcup_{i=1}^{N} \mathcal{W}_{i}^{\delta}
$$

and for every $i \in \mathcal{N}$ there exists at least one index $j \neq$ $i \in \mathcal{N}$ such that

$$
\operatorname{Int}\left\{\mathcal{W}_{i}^{\delta} \cap \mathcal{W}_{j}^{\delta}\right\} \neq \emptyset,
$$


where $\operatorname{Int}\{\cdot\}$ denotes the set interior operator. By considering the output admissible set for the generic $C G_{i}$,

$$
\begin{aligned}
Z_{i}^{\delta}:=\left\{\left[r^{T}, x^{T}\right]^{T} \in\right. & \mathbb{R}^{m} \times \mathbb{R}^{n} \mid \\
& \left.c_{i}(k, x, r) \in \mathcal{C}, \forall k \in \mathbb{Z}_{+}\right\},
\end{aligned}
$$

the set of all states which can be steered to feasible equilibrium points without constraint violation as

$\mathcal{X}_{i}^{\delta}:=\left\{x \in \mathbb{R}^{n} \mid\left[\begin{array}{c}w \\ x\end{array}\right] \in Z_{i}^{\delta}\right.$ for at least one $\left.w \in \mathbb{R}^{m}\right\}$,

$i \in \mathcal{N}$, we have that, in view of (18), the following condition holds:

$$
\operatorname{Int}\left\{\mathcal{X}_{i}^{\delta} \cap \mathcal{X}_{j}^{\delta}\right\} \neq \emptyset, \quad i, j \in \mathcal{N} .
$$

A convenient transition reference $\hat{r} \in \operatorname{Int}\left\{\mathcal{W}_{i}^{\delta} \cap\right.$ $\left.\mathcal{W}_{j}^{\delta}\right\}$, with $\hat{x} \in \operatorname{Int}\left\{\mathcal{X}_{i}^{\delta} \cap \mathcal{X}_{j}^{\delta}\right\}$ being the equilibrium steady-state corresponding to $\hat{r}$, can be defined such that $\left[\hat{r}^{T}, \hat{x}^{T}\right]^{T} \in Z_{i}^{\delta} \cap Z_{j}^{\delta}$. Assume that $C G_{i}$ unit is in use at $t=\bar{t}, r(\bar{t}) \in \mathcal{W}_{i}^{\delta}, r(\bar{t}+1) \in \mathcal{W}_{j}^{\delta}$ and the condition $\mathcal{W}_{i}^{\delta} \cap \mathcal{W}_{j}^{\delta} \neq \emptyset$ holds true; then an HCG scheme can be adopted according to the following switching logic:

\section{Switching procedure}

1. If the distance between the equilibrium $x_{i}^{e q}$ and the actual state $x(t)$ is minimal, the supervisor solves and applies

$$
\begin{aligned}
g(\bar{t}+k):=\arg \min _{w \in \mathcal{V}_{i}(x(\bar{t}+k))}\|w-r(\bar{t})\|_{\Psi}, \\
k=1, \ldots, \bar{k} .
\end{aligned}
$$

2. At $t=\bar{t}+\bar{k}$, as soon as $x(t) \in \operatorname{Int}\left\{\mathcal{X}_{i}^{\delta} \cap \mathcal{X}_{j}^{\delta}\right\}$ and

$$
j:=\arg \min _{k}\left\|x_{k}^{e q}-x(t)\right\|,
$$

the supervisor switches to $C G_{j}$ and solves

$$
\begin{array}{r}
g(\bar{t}+k):=\arg \min _{w \in \mathcal{V}_{j}(x(t))}\|w-r(\bar{t}+1)\|_{\Psi}, \\
t \geq \bar{t}+\bar{k}+1 .
\end{array}
$$

4.2. Time-varying constraints. Let us consider $L$ different constraint scenarios, denoted by $\mathcal{C}_{j}$, where $j \in$ $\mathcal{J}:=\{1,2, \ldots, L\}$, and introduce the following sets doubly indexed with respect to to the current couple equilibrium/constraints scenario:

$$
\mathcal{W}_{(\bullet, j)}^{\delta}:=\left\{w \in \mathbb{R}^{m}: \bar{c}_{w} \in \mathcal{C}_{j}^{\delta}\right\}, \quad \forall j \in \mathcal{J},
$$

where $\mathcal{W}_{(\bullet, j)}^{\delta}$ (the bullet denotes a fixed equilibrium configuration) is the set of all commands $w$ whose steady-state evolutions of $c$ satisfy the $j$-th constraint configuration $\mathcal{C}_{j}$ with a tolerance margin $\delta$. Assume $\mathcal{W}_{(\bullet, j)}^{\delta} \neq \emptyset, \forall j \in \mathcal{J}$ and $\mathcal{C}_{j}^{\delta} \neq \emptyset$, moreover $\mathcal{W}_{(\bullet, j)}^{\delta}$ satisfies the set overlapping property: Let $\left(j_{1}, j_{2}\right) \in \mathcal{J}$. Then

$$
\mathcal{C}_{j_{1}}^{\delta} \bigcap \mathcal{C}_{j_{2}}^{\delta} \neq \emptyset \Leftrightarrow \mathcal{W}_{\left(\bullet, j_{1}\right)}^{\delta} \bigcap \mathcal{W}_{\left(\bullet, j_{2}\right)}^{\delta} \neq \emptyset .
$$

The next definitions are then instrumental to characterize all the possible switching features of the sets $\mathcal{C}_{j}^{\delta}$ and $\mathcal{W}_{(\bullet, j)}^{\delta}$.

Definition 1. The state $x \in \mathbb{R}^{n}$ is $\mathcal{C}_{j}^{\delta}$-admissible, $j \in \mathcal{J}$, if there exists $w \in \mathcal{W}_{(\bullet, j)}^{\delta}$ such that $c(k, x, w) \in \mathcal{C}_{j}^{\delta}, \forall k \in$ $\mathbb{Z}_{+}$. The pair $(x, w)$ is said to be $\mathcal{C}_{j}^{\boldsymbol{\delta}}$-executable.

Definition 2. Let $x \in \mathbb{R}^{n}$ be a state $\mathcal{C}_{\mathbf{j}^{-}}^{\delta}$-admissible, $j^{-} \in \mathcal{J}$, and $\mathcal{C}_{j^{+}}^{\delta}, j^{+} \neq j^{-}$, a constraint configuration to be fulfilled at future time instants. The state $x$ is switching- $\mathcal{C}_{\boldsymbol{j}^{-}}^{\boldsymbol{\delta}}$-admissible if there exists $w \in \mathcal{W}_{\left(\bullet, j^{-}\right)}^{\delta}$ such that $c(k, x, w) \in \mathcal{C}_{j^{+}}^{\delta}, \forall k \in \mathbb{Z}_{+}$. The pair $(x, w)$ is said to be switching- $\mathcal{C}_{j^{-}}^{\delta}$-executable and the constraint configuration $\mathcal{C}_{j^{+}}^{\delta}$ switchable.

Moreover,

$\mathcal{V}_{(\bullet, j)}(x):=\left\{w \in \mathcal{W}_{(\bullet, j)}^{\delta}: c(k, x, w) \in \mathcal{C}_{j}^{\delta}, \forall k \in \mathbb{Z}_{+}\right\}$

$i, j \in \mathcal{I}$, represent the sets of all constant virtual sequences in $\mathcal{W}_{(\bullet, j)}^{\delta}$ whose $c$-evolutions, starting from a $\mathcal{C}_{j}^{\delta}$-admissible state $x$, satisfy the prescribed constraint configuration $\mathcal{C}_{j}^{\delta}$ also during transients. In consequence, for a fixed $j \in \mathcal{J}$, we have $\mathcal{V}_{(\bullet, j)}(x) \subset \mathcal{W}_{(\bullet, j)}^{\delta}$. Then, whenever the supervisory unit selects the CG candidate with respect to the $j$-th constraints configuration $\left(C G_{(\bullet, j)}\right)$, a command $g(t)$ is computed as a solution to the following constrained optimization problem:

$$
g(t)=\arg \min _{w \in \mathcal{V}_{(\bullet, j)}(x(t))}\|w-r(t)\|_{\Psi} .
$$

An admissible HCG strategy can then be developed if at each switching instant $\bar{t}$, chosen by the supervisory unit, the current state $x(\bar{t})$ is switching-admissible. The following sets:

$$
\begin{aligned}
\mathcal{X}_{(\bullet, j)}^{\delta}:= & \left\{x \in \mathbb{R}^{n}: c(k, x, w) \in \mathcal{C}_{j}^{\delta},\right. \\
& \text { for at least one } w \in \mathcal{W}_{(\bullet, j)}^{\delta}, \\
& \left.\forall k \in \mathbb{Z}_{+}\right\}, \quad \forall j \in \mathcal{J},
\end{aligned}
$$

are finally introduced to characterize all the states $\mathcal{C}_{j}^{\delta}$-admissible (each state $x \in \mathcal{X}_{(\bullet, j)}^{\delta}$ can be steered to an equilibrium point without constraint violation). 
Remark 1. Note that even if the initial and final set-points belong to $\mathcal{W}_{(i, j)}^{\delta}$, or the final set-point is fixed in time, the $i$-th linearized model approximates the nonlinear dynamics (13) only in a neighbouring region of the actual $i$-th equilibrium point (see, e.g., Khalil, 1996). While the state evolution departs in fact significantly from $x_{i}^{e q}$, an obvious performance degradation will take place and a possible way to cope with this drawback is to consider the opportunity to commute the controller structure also when the distance between the current state $x(t)$ and the actual equilibrium point $x_{i}^{e q}$ significantly increases. To this end, let $\mathcal{T}_{i} \subset \mathbb{R}^{n}$ be the set of states such that the $i$-th linearized model retains its validity in terms of nonlinear system trajectory approximation, and let $\bar{t}$ be the actual instant when using $C G_{(i, j)}$. Then, if $x(\bar{t}) \notin \mathcal{T}_{i}$ a switching amongst the CG candidates takes place by means of the Switching procedure. The set $\mathcal{T}_{i}$ can be obtained by resorting to linearization arguments, see (14)-15), in terms of semi-algebraic conditions. By noting that an equivalent description of this set of states can be given by deriving an appropriate normalized polynomial level surface function $V(z)$ in the extended space $z:=\left[\delta x_{p}^{T}, \delta u^{T}\right]^{T}$, i.e.,

$$
\mathcal{T}_{i}:=\operatorname{Proj}_{X}\left\{z \in \mathbb{R}^{n+m} \mid V_{i}(z) \leq 1\right\},
$$

where $\operatorname{Proj}_{X}$ is the projection onto $X$ by exploiting the requirements (15), there exists a polynomial level surface function $V_{i}(z)$ for the semi-algebraic set $\{z \in$ $\left.\mathbb{R}^{n+m} \mid V_{i}(z) \leq 1\right\}$ if the following set inclusion holds:

$$
\begin{gathered}
\left\{z \in \mathbb{R}^{n+m} \mid V_{i}(z) \leq 1\right\} \cap\left\{z \in \mathbb{R}^{n+m} \mid z^{T} z \leq r^{i^{2}}\right\} \\
\subseteq\left\{z \in \mathbb{R}^{n+m} \mid F_{i}(z)^{T} F_{i}(z) \leq \gamma^{i^{2}}\right\}
\end{gathered}
$$

The computation of $\mathcal{T}_{i}$ can be achieved by solving a sum-of-squares optimization problem (see Tan and Packard, 2008).

\section{Real-time hybrid command governor scheme}

In this section a supervisory CG-based real-time scheme for the proposed architecture is detailed. Given a set of operating points $\left\{\left(x_{i}^{e q}, u_{i}^{e q}\right)\right\}_{i=1}^{N}$ for (13), we will assume that at each time instant $t$ the supervisory unit is informed on the current plant structure; the time interval $T_{O N}$, necessary for the on-line computation of the CG action, is such that $T_{O N}<T_{s}$, with $T_{s}$ being the sampling time. follows.

The idea we want to develop can be summarized as

RT-HCG strategy. At the generic time instant $t$, the plant is under the action of the $C G_{(i, j)}$ unit and the supervisor receives the information on the plant structure to be fulfilled at $t+1$. The supervisor logic retains valid the
$C G_{(i, j)}$ unit as long as the distance between the equilibrium $x_{i}^{e q}$ and the actual state $x(t)$ is minimal and the constraint configuration is unchanged. The supervisor switches according to

- set-point change: the selected unit is $C G_{\left(i^{\prime}, j\right)}$ unit where $i^{\prime}$ is chosen according to the rule

$$
i^{\prime}:=\arg \min _{k}\left\|x_{k}^{e q}-x(t)\right\|
$$

- constraints change: the selected unit is $C G_{\left(i, j^{\prime}\right)}$ if a constraint configuration $\mathcal{C}_{j^{\prime}} \neq \mathcal{C}_{j}$ occurs.

The supervisor scheme is depicted in Fig. 3. $r(t \mid t+$ 1 ) is the reference known at the time instant $t$ and to be tracked at $t+1, \mathcal{C}_{t, t+1}$ is the constraint configuration known at $t$ and to be fulfilled at $t+1$, and $r(t), x(t)$ are the reference and state measurements. The following distinct events may occur:

- Set-point change: If $r(t \mid t+1) \notin \mathcal{W}_{(i, j)}^{\delta}$, a switching to the $i^{\prime}$-th model selected by (27) must be imposed.

- Constraint configuration change: Because the on-line design of the $C G_{\left(i^{\prime}, j\right)}$ unit could require more than one sampling time and the action of $C G_{(i, j)}$ is no longer admissible, to guarantee constraint fulfilment at each time instant $t$ an adequate controller must be considered. Such a regulator, in place of the primal control law $K_{i}$ and the $C G_{(i, j)}$ device, should be capable to satisfy all the constraints regardless set-point tracking properties until the $C G_{\left(i^{\prime}, j\right)}$ computation phase is accomplished. Hereafter, we denote it as the safe controller $K_{\text {safe }}$.

- Equilibrium change: By checking (20) it results that a switching to the $i^{\prime}$-th model is more adequate to approximate the plant behavior (13).

The Supervisor behaviour is described by a finite state automaton with three operating states:

- HOME: normal operating condition under a CG unit action,

- EQ-SW: handling of a set-point or an equilibrium point change event,

- CNF-SW: handling of a constraint configuration change event.

The Supervisor is set by default to the HOME state where the control action is carried out by a single periodic task $\tau_{C G_{(i, j)}}$ which runs at the highest priority level and executes all the CG operations. In particular, the $\tau_{C G_{(i, j)}}$ actions are reference and state measurements acquisition, 


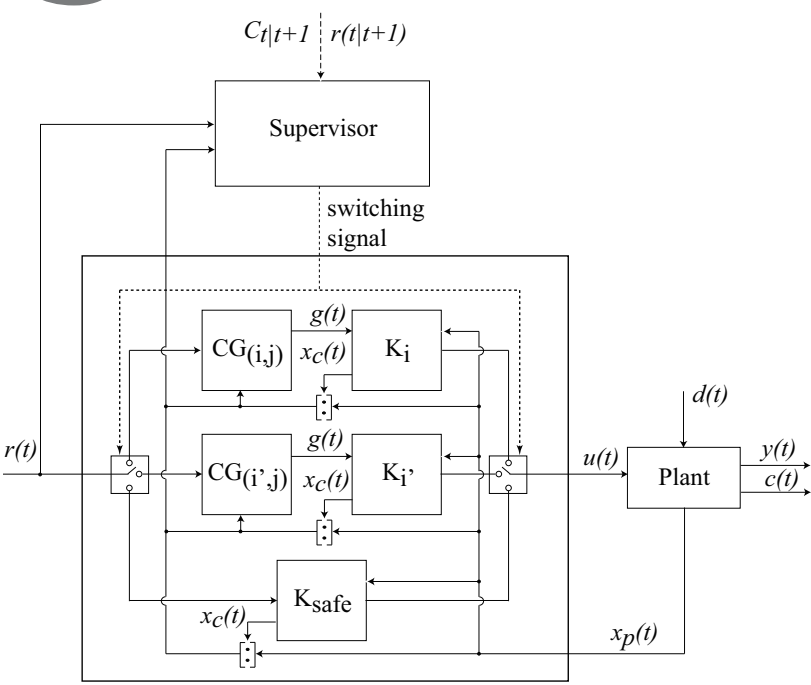

Fig. 3. Supervisory scheme.

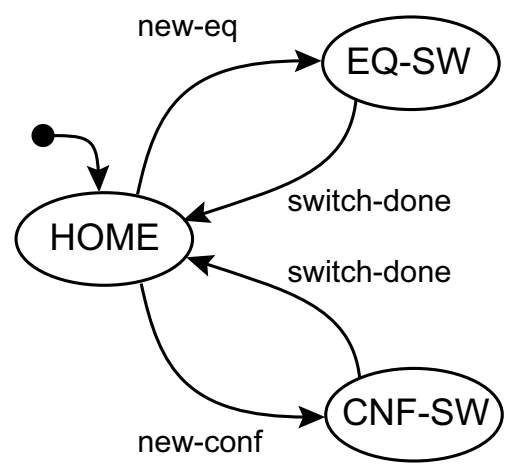

Fig. 4. Supervisor automaton.

on-line computation of the reference governor output $g(t)$, primal controller execution and application of $g(t)$.

If the set-point or an equilibrium point change occurrence is detected (new-eq event), the Supervisor switches to the EQ-SW state where the operation mode enables the computation of the new CG design. In particular, the transition HOME $\rightarrow$ EQ-SW occurs when one of the following two events take place: $x(t) \notin \mathcal{T}_{i}$ or $r(t \mid t+1) \neq r(t)$ and $r(t \mid t+1) \notin \mathcal{W}_{(i, j)}^{\delta}$.

Then

$$
i^{\prime}=\arg \min _{k}\left\|x_{k}^{e q}-x(t)\right\|, \quad i^{\prime} \neq i,
$$

and $C G_{\left(i^{\prime}, j\right)}$ is designed under the condition $\operatorname{Int}\left\{\mathcal{W}_{(i, j)}^{\delta} \cap \mathcal{W}_{\left(i^{\prime}, j\right)}^{\delta}\right\} \neq \emptyset$.

From a real-time perspective, the design of the CG is assigned to an aperiodic task $\tau_{S W}$ which is released when EQ-SW becomes active and runs at a lower priority level with respect to $\tau_{C G_{(i, j)}}$. In the general case, $\tau_{S W}$ is not able to complete its task (CG design) within a single sampling period because a fraction of this time interval must be used for the execution of $\tau_{C G_{(i, j)}}$. Therefore, the process $\tau_{S W}$ is pre-empted a certain number of times by $\tau_{C G_{(i, j)}}$. As soon as the task $\tau_{S W}$ accomplishes its job (switch-done event), the Supervisor switches to new $C G_{\left(i^{\prime}, j\right)}$, and the system operation mode is set to HOME.

A different mode transition occurs when a constraint configuration change is detected (new-conf event), i.e., HOME $\rightarrow$ CNF-SW, where the one-step ahead constraint configuration is such that $\mathcal{C}_{j^{\prime}}=: \mathcal{C}_{t \mid t+1} \nsubseteq \mathcal{C}_{j}$, and, due to (22), we have that $\mathcal{C}_{t \mid t+1} \cap \mathcal{C}_{j} \neq \emptyset$. In this case, the CG design is accomplished by an aperiodic task instance $\tau_{S W}$. On the other hand, at the actual time instant $t$, the actions provided by $\tau_{C G_{(i, j)}}$ are not adequate because the fulfilment of the new constraint set is no longer guaranteed. Then, within $[t, t+1]$ the Supervisor establishes the execution of a new task $\tau_{C S}$ whose actions are computation of $K_{\text {safe }}$ and its application in order to ensure at least constraint satisfaction. At each future sampling time $[t+i, t+1+i], i \geq 1$, a periodic task $\tau_{\text {safe }}$ applies the control action due to $K_{\text {safe }}$. Note that both $\tau_{C S}$ and $\tau_{\text {safe }}$ tasks inherit the priority level of $\tau_{C G_{(i, j)}}$. Finally, when $\tau_{S W}$ (switch-done event) ends, the Supervisor de-schedules $\tau_{\text {safe. }}$. The task $\tau_{C G_{\left(i, j^{\prime}\right)}}$ is now restarted and equipped with the new on-line computed CG. The system operation mode is then reset to the HOME state.

The main properties of the RT-HCG strategy are summarized in the following proposition.

\section{Proposition 1. Suppose that}

- (11) is asymptotically stable and offset-free;

- at each time instant $t$, the supervisory unit is informed on the current plant structure and $T_{O N}<T_{s}$;

- (17)-(18) hold for the time-varying set-point scenario;

- the property (22) is valid for the time-varying constraint scenario.

Then

- no constraint configuration change occurrences: all the properties of the CG device (see Bacconi et al., 2007, Theorem 1, p. 345) are preserved;

- constraint configuration change occurrences: the $K_{\text {safe }}$ controller guarantees "plant workability": asymptotic stability and constraint fulfilment.

Proof. Under no constraint configuration change, the overlapping condition (18) directly guarantees the viability property by resorting to similar arguments as those by Bemporad et al. (1997). During a constraint configuration change, its fulfilment and stability are never 
lost due to the controller $K_{\text {safe }}$. On the contrary, tracking performance may be lost because the system behaves essentially in an open-loop fashion. Finally, because this phase lasts for a finite time period, tracking operations can be safely recovered by connecting the CG unit associated with the new constraint configuration.

\section{Simulations}

The aim of this section is to demonstrate the effectiveness both in terms of reconfiguration capabilities and control performance of the proposed RT-HCG strategy by means of numerical simulations on nonlinear aircraft models. All computations were carried out on a PC Intel Quad Core with the Matlab LMI and Optimization Toolboxes.

6.1. HAPD: An unexpected manoeuvre with command saturation. The benefits of the proposed reconfiguration strategy were verified by using the full nonlinear HAPD aircraft model including 25 symmetrical and 25 asymmetrical flexible modes, sensors and actuators dynamics. Longitudinal dynamics were excited by means of the following pitch angle manoeuvre and flight scenario.

Doublet on the pitch angle demand $\left(\boldsymbol{\theta}_{\text {ref }}\right)$. At steady state wing levelled forward flight conditions at altitude $h_{0}=500 \mathrm{~m}$ and true air speed $V_{0}=20 \mathrm{~m} / \mathrm{s}$, at $t=1 \mathrm{~s}$ a pitch angle command of $20 \mathrm{deg}$ is first given for a duration of $2 \mathrm{~s}$; then, a doublet reference command of $10 \mathrm{deg}$ is given within the time interval $[5,11]$ s (see the dashed line in Fig. 5) .

For RT-HCG design purposes, the following assumptions were made: actuator and sensor dynamics were considered negligible; by resorting to well-known residual stiffness techniques, aeroelastic dynamics were assumed to be instantaneous. Moreover, two levelled forward flight conditions, corresponding to two equilibrium conditions of the $6 \mathrm{DoF}$ nonlinear aircraft model (1)-(8), are considered:

- $\left(\right.$ altitude $\left.=50.0 \mathrm{~m}, V_{0}=17 \mathrm{~m} / \mathrm{s}\right) \rightarrow$

$$
\left\{\begin{aligned}
x_{1}^{\mathrm{eq}}= & {[17,2.38,0,0,0,0,0,2.38]^{T}, } \\
u_{1}^{\mathrm{eq}}= & {[4.74,4.74,4.74,4.74,4.74,4.74,} \\
& 0,0,0,0,0,0]^{T},
\end{aligned}\right.
$$

- $\left(\right.$ altitude $\left.=500 \mathrm{~m}, V_{0}=23 \mathrm{~m} / \mathrm{s}\right) \rightarrow$

$$
\left\{\begin{aligned}
x_{2}^{\mathrm{eq}}= & {[23,-1.56,0,0,0,0,0,-1.56]^{T} } \\
u_{2}^{\mathrm{eq}}= & {[7.6,7.6,7.6,7.6,7.6,7.6} \\
& 0,0,0,0,0,0]^{T}
\end{aligned}\right.
$$

where

$$
x(t)=[V(t), \alpha(t), \beta(t), p(t), q(t), r(t), \phi(t), \theta(t)]^{T}
$$

and

$$
u(t)=\left[\begin{array}{l}
\text { ElevatorIB }-D X(t) \\
\text { ElevatorIB }-S X(t) \\
\text { ElevatorMID }-D X(t) \\
\text { ElevatorMID }-S X(t) \\
\text { ElevatorOB }-D X(t) \\
\text { ElevatorOB }-S X(t) \\
\text { AileronIB }-D X(t) \\
\text { AileronIB }-S X(t) \\
\text { AileronOB }-D X(t) \\
\text { AileronOB }-S X(t) \\
\text { RudderSUP }(t) \\
\text { RudderINF }(t)
\end{array}\right] .
$$

The linearized models obtained in correspondence with the above equilibrium conditions were discretized using the forward Euler difference scheme with a sampling time $T_{s}=0.01 \mathrm{~s}$ and used for the implementation of the proposed predictive strategy.

The main numerical results are collected in Figs. 547 As highlighted in Fig. 5 the HCG device outperforms the single $C G_{(1,1)}$ action when the tracking capabilities on the pitch angle are considered. This is clearly achieved by means of the CG switchings. In fact, the $C G_{(1,1)} \rightarrow$ $C G_{(2,1)}$ switching occurring at $t=1.3 \mathrm{~s}$ and at $t=9.4 \mathrm{~s}$ have the merit to enforce the elevators control action (see Fig. 6) and, in consequence, the overall tracking performance.

The minimum/maximum computational times to generate commands in one step are summarized in Table 3 From the analysis of this a table, it can be noticed that the sampling time $\left(T_{s}=0.01 \mathrm{~s}\right)$ is non-critical because all the computational times are less than the sampling period.

Table 3. Computational loads (ms).

\begin{tabular}{|c|c|c|}
\hline State & Min. & Max. \\
\hline \hline HOME & 0.4 & 0.8 \\
\hline EQ-SW & 2.18 & 5.3 \\
\hline
\end{tabular}

6.2. P92: HCG performance in the presence of actuator reduced capabilities. The flight control system design of a P92 small commercial aircraft subject to constraints on the control surface positions and their rates of variations is considered. A full nonlinear simulator in the Matlab/Simulink environment was used. The external atmospheric disturbances are generated by resorting to a Von Karman continuous time wind turbulence model implemented on the basis of the mathematical representation in the military specification MIL-F-8785C. The following roll rate manoeuvre was in simulation.

Doublet on the roll rate demand ( $\left.\mathrm{p}_{\mathrm{ref}}\right)$. Assuming an 


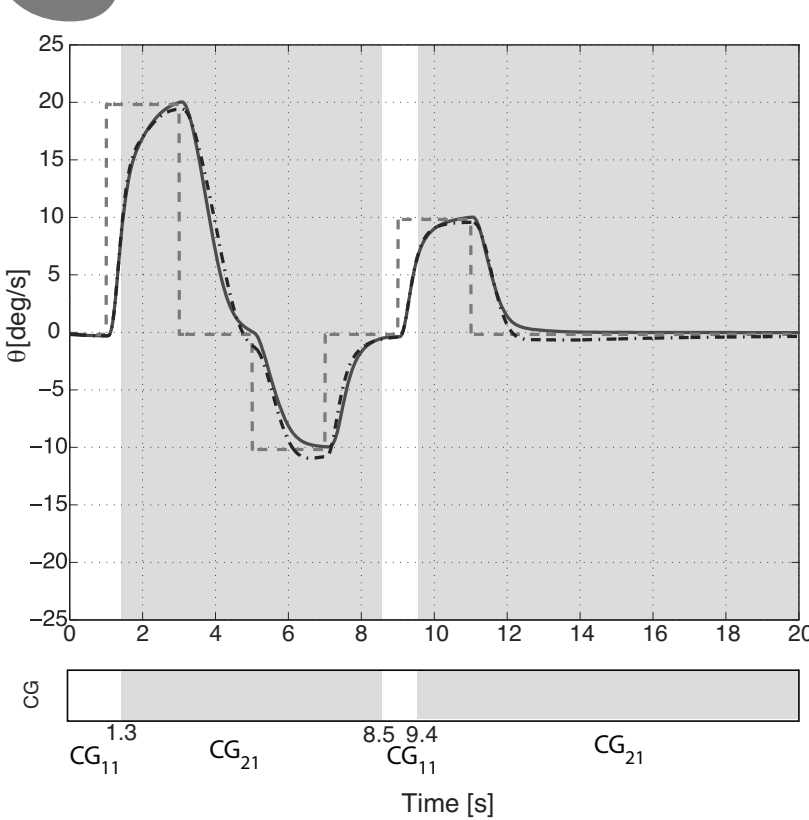

Fig. 5. RT-HCG (continuous line), $C G_{(1,1)}$ (dashed-dotted line). The dashed line is the reference signal $\theta_{\text {ref }}$.
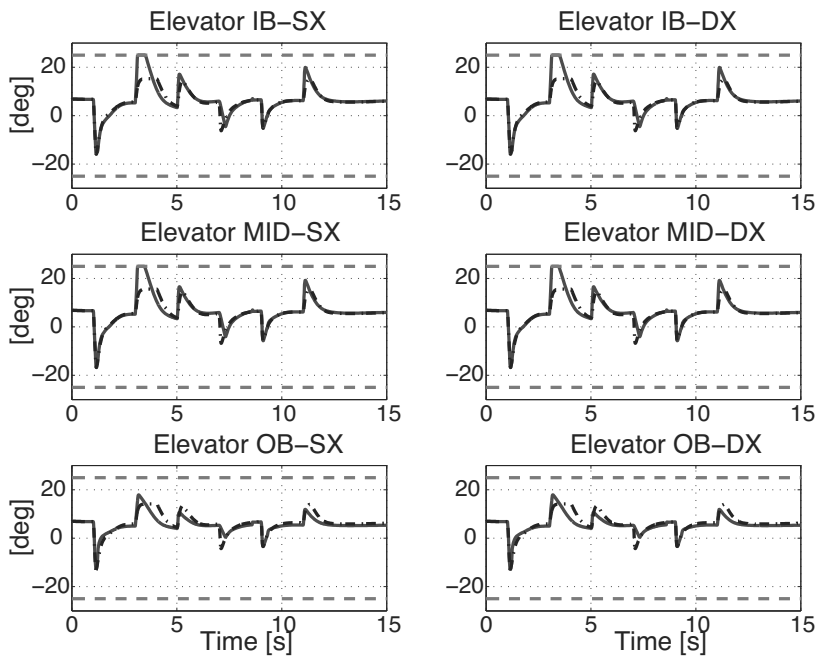

Fig. 6. Elevators control effort. The dashed lines represent the prescribed constraints.

initial steady state wing levelled forward flight at the altitude $h_{0}=2000 \mathrm{~m}$ and $V_{0}=45 \mathrm{~m} / \mathrm{s}$ at $t=1 \mathrm{~s} \mathrm{a}$ reference of $30 \mathrm{deg} / \mathrm{s}$ is requested for a duration of $2 \mathrm{~s}$. Then, at $t=5 \mathrm{~s}$ a reference of $-30 \mathrm{deg} / \mathrm{s}$ is requested for the same duration. Moreover, in the time interval from $t=2 \mathrm{~s}$ to $t=8 \mathrm{~s}$, a failure occurs modelled as a decreased maximum surface deflection from $30 \mathrm{deg}$ to $8 \mathrm{deg}$, which implies a reduction of more than $70 \%$ of the aerodynamic surfaces control capabilities. A moderate atmospheric turbulence with a maximum wind speed of about 4 $\mathrm{m} / \mathrm{s}$ and standard deviation of about $1 \mathrm{~m} / \mathrm{s}$ is considered.
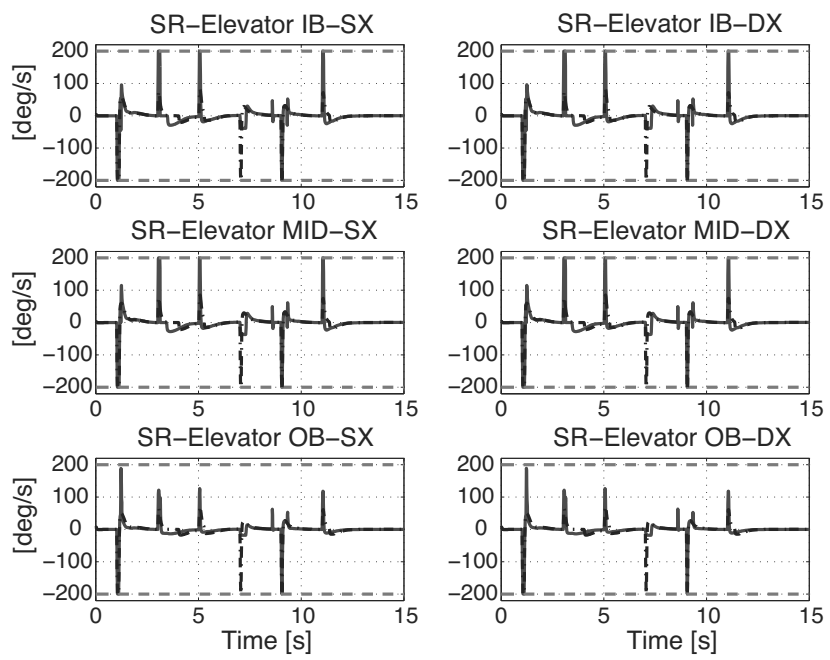

Fig. 7. Rates of variation on elevator deflections. The dashed lines represent the prescribed constraints.

The relevant numerical results are depicted in Figs. 814 The roll angle $\phi$ increase, due to the roll rate request $p_{\text {ref }}$ (Fig. 10), implies that the side-slip angle $\beta$ increases too in response to a lateral velocity, see Fig. 9. In response to this behaviour, a yaw rate is induced in order to align the aircraft with the airspeed direction, while the elevator is used to restore the flight speed and attitude, see Fig. 12.

Figure 8 also provides the tracking performance under both faulty (grey zone) and healthy conditions (the $p$ graph). As expected, the malfunction occurrence gives rise to unavoidable loss of tracking capabilities. Note also that the $p$ response discrepancy between the healthy and faulty RT-HCG behaviours evidently arises when the saturation occurs (see Fig. 11 at about $6 \mathrm{~s}$ ) because, until this event, the constraint structure change cannot influence the aircraft dynamics.

\section{Conclusions}

In this paper, an analysis on the applicability and effectiveness of a recent developed hybrid control CG-based scheme was presented. As one of its main merits, by resorting to a supervisory framework this strategy is able to quickly adapt to unexpected plant structure changes and at the same time to guarantee constraint fulfilment and tracking capabilities.

Simulation studies were devoted to investigating the performance of the RT-HCG scheme by using the HAPD and P92 test-bed aircraft subject to both the position and rate of variation limitation and anomalous dynamical behaviours. The numerical results have shown the effectiveness of the proposed approach both in terms of constraints satisfaction and control reconfiguration 

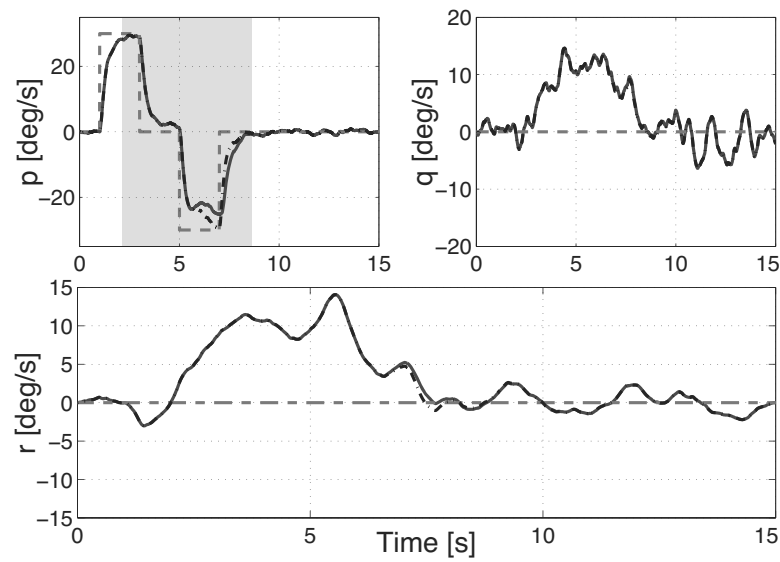

Fig. 8. Faulty RT-HCG response (continuous line), healthy RTHCG response (dashed-dotted line). The dashed line is the reference signal. The grey zone indicates faulty conditions.
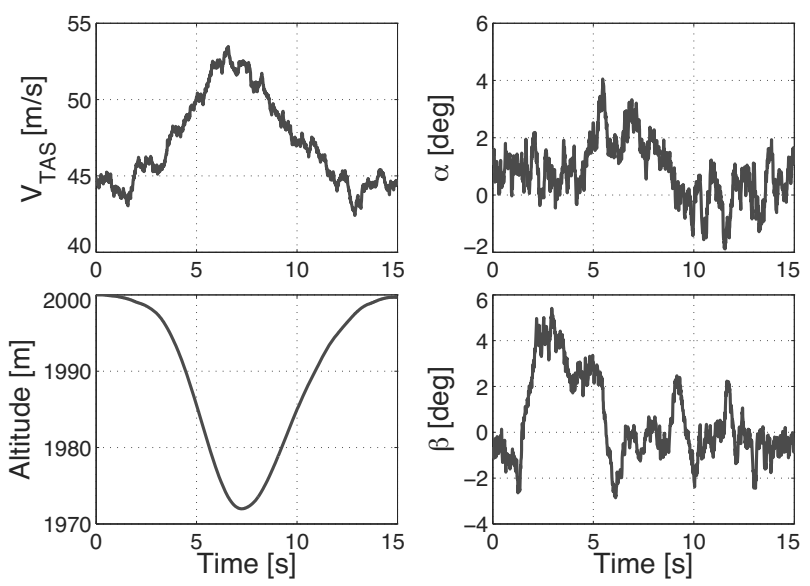

Fig. 9. True air speed, altitude, angle-of-attack and sideslip angle dynamical behaviours.
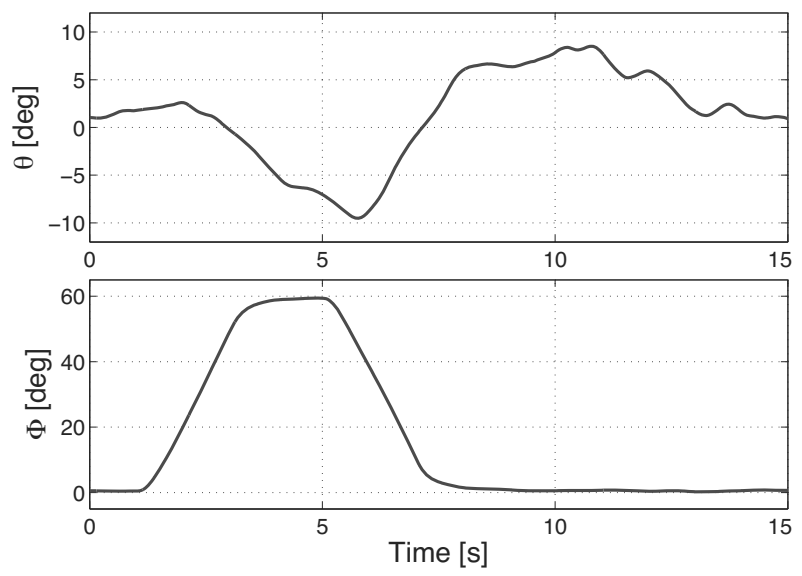

Fig. 10. Pitch angle and roll angle dynamical behaviours.

capabilities.

Future developments will aim to relax the hypothesis that the supervisor exactly knows the reference/constraint

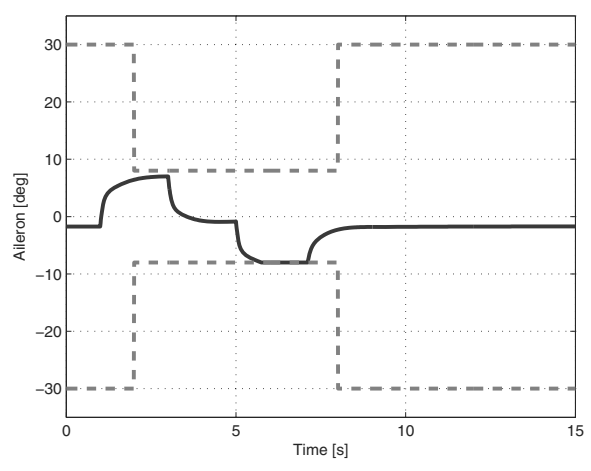

Fig. 11. Ailerons control effort. The dashed lines represent the prescribed constraints.

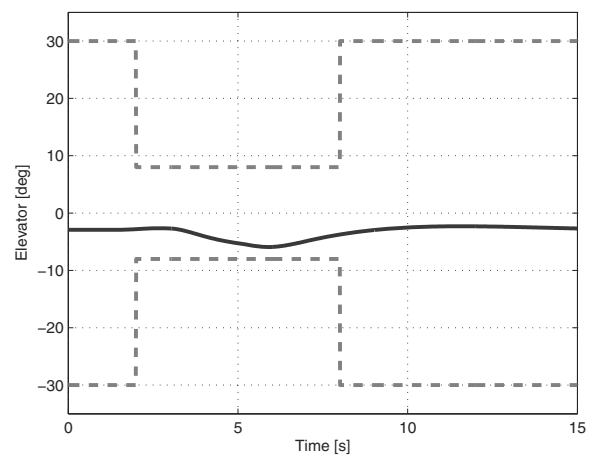

Fig. 12. Elevators control effort. The dashed lines represent the prescribed constraints.

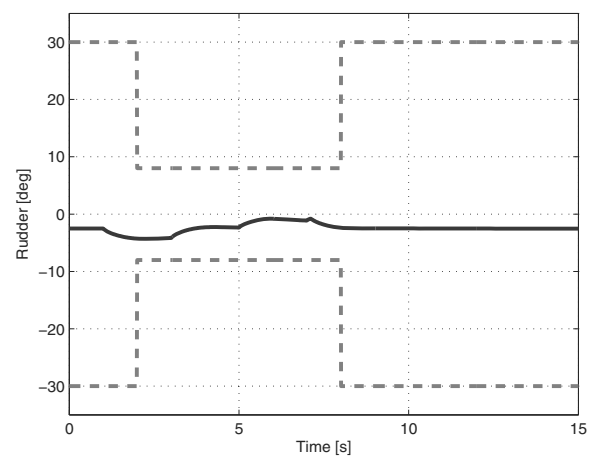

Fig. 13. Rudders control effort. The dashed lines represent the prescribed constraints.

configuration to be fulfilled at the next time instant. The latter prescribes that robust (against model uncertainties and disturbance/noise effects) model estimator units should be properly designed so that the correct plant structure is promptly and safely detected. Moreover, a further research line will address the generalization of the fault description in order to cover more severe scenarios, e.g., stuck fault occurrences. 


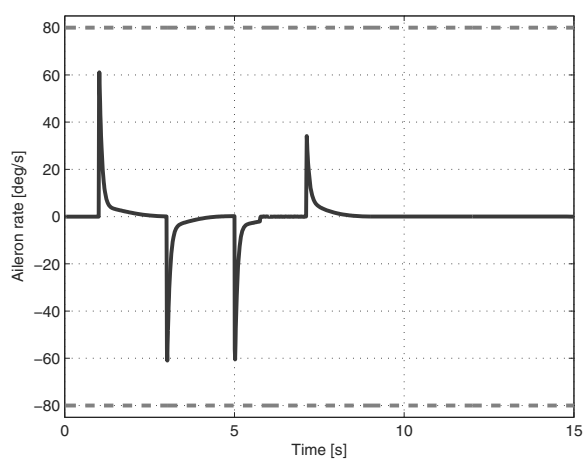

Fig. 14. Rates of variation on aileron deflections. The dashed lines represent the prescribed constraints.

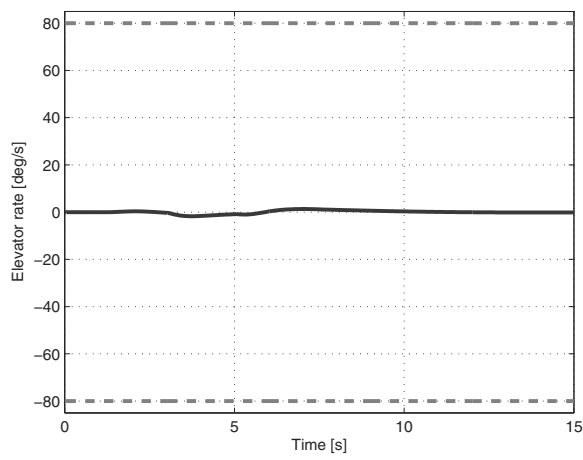

Fig. 15. Rates of variation on elevator deflections. The dashed lines represent the prescribed constraints.

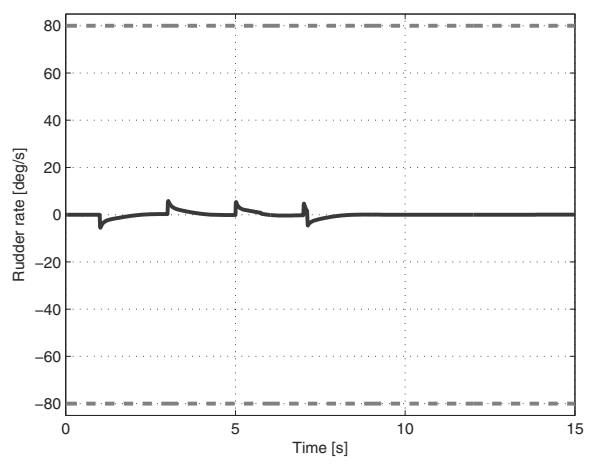

Fig. 16. Rates of variation on rudder deflections. The dashed lines represent the prescribed constraints.

\section{References}

Angeli, D., and Mosca, E. (1999). Command governors for constrained nonlinear systems, IEEE Transactions on Automatic Control 44(4): 816-820.

Angeli, D., Casavola, A. and Mosca, E. (2001). On feasible set-membership state estimators in constrained command governor control, Automatica 37(1): 151-156.

Bacconi, F., Mosca, E. and Casavola, A. (2007). Hybrid constrained formation flying control of micro-satellites,
IET Control Theory Applications 1(2): 513-521.

Bemporad, A., Casavola, A. and Mosca, E. (1997). Nonlinear control of constrained linear systems via predictive reference management, IEEE Transactions on Automatic Control 42(3): 340-349.

Bemporad, A. (1998). Reference governor for constrained nonlinear systems, IEEE Transactions on Automatic Control 43(3): 415-419.

Blackford, L.S., Demmel, J., Dongarra, J., Duff, I., Hammarling, S., Henry, G., Heroux, M., Kaufman, L., Lumsdaine, A., Petitet, A., Pozo, R., Remington, K. and Whaley R.C. (2003). An updated set of basic linear algebra subprograms (BLAS), ACM Transactions on Mathematical Software 28(2): 135-151.

Blanke, M., Kinnaert, M., Lunze, J. and Staroswiecki, M. (2006). Diagnosis and Fault Tolerant Control, Springer-Verlag, Berlin/Heidelberg.

Branicky, M.S. (1998). Multiple Lyapunov functions and other analysis tools for switched and hybrid systems, IEEE Transactions on Automatic Control 43(4): 475-482.

Chen, S.H., Tao, G., and Joshi, S.M. (2002). On matching conditions for adaptive state tracking control of systems with actuator failures, IEEE Transactions on Automatic Control 47(3): 473-478.

Famularo, D., Franzè, G., Furfaro, A. and Mattei, M. (2011). A hybrid real-time supervisory scheme for nonlinear systems, Proceedings of the 2011 American Control Conference, ACC 2011, San Francisco CA, USA, pp. 305-310.

Franzè, G., Furfaro, A., Mattei, M. and Scordamaglia, V. (2013). An hybrid command governor supervisory scheme for flight control systems subject to unpredictable anomalies, Proceedings of the 2nd International Conference on Control and Fault-Tolerant Systems, Nice, France, (CD-ROM).

Gao, Z. and Antsaklis, P.K. (1991). Stability of the pseudo-inverse method for reconfigurable control systems, International Journal of Control 53(3): 717-729.

Garone, E., Tedesco, F., and Casavola, A. (2010). A feed-forward command governor strategy for constrained linear systems, Proceedings of Nolcos 2010, Bologna, Italy.

Gilbert, E.G., Kolmanovsky, I. and Tin Tan, K. (1995). Discrete-time reference governors and the nonlinear control of systems with state and control constraint, International Journal of Robust and Nonlinear Control 5(5): 487-504.

Gilbert, E.G. and Kolmanovsky, I. (1999). Fast reference governors for systems with state and control constraint and disturbance inputs, International Journal of Robust and Nonlinear Control 9(15): 1117-1141.

Guo, C. and Song, Q. (1999). Real-time control of variable air volume system based on a robust neural network assisted PI controller, IEEE Transactions on Control Systems Technology 17(3): 600-607.

Iserman, R.. and Ballè, P. (1997). Trends in the application of model-based fault detection and diagnosis of technical processes, Control Engineering Practice 5(5): 709-719. 
Khalil, H.K. (1996). Nonlinear Systems, Prentice Hall, Upper Saddle River, NJ.

Magree, D., Yucelen, T., and Johnson, E.N. (2012). Command governor-based adaptive control of an autonomous helicopter, Proceedings of the AIAA Conference, Minneapolis, MI, USA, pp. 1-13.

Mattei, M., Famularo, D. and Labate, C.V. (2013). A constrained control strategy for the shape control in thermonuclear fusion tokamaks, Automatica 49(1): 169-177.

Mhaskar, P., McFall, C., Gani, A., Christofides, P.D., and Davis, J. F. (2008). Isolation and handling of actuator faults in nonlinear systems, Automatica 44(1): 53-62.

Micksh, T., Gambier A. and Badreddin, E. (2008). Real-time implementation of fault-tolerant control using model predictive control, Proceedings of the 17th IFAC World Congress, Seoul, Korea, pp. 11136-11141.

Park, S.J. and Yang, J.M. (2009). Supervisory control for real-time scheduling of periodic and sporadic tasks with resource constraint, Automatica 45(11): 2597-2604.

Patton, R.J. (1997). Real-time implementation of fault-tolerant control using model predictive control, Proceedings of the 3rd IFAC Symposium on Fault Detection, Supervision and Safety for Technical Processes, Hull, UK, pp. 1033-1055.

Scordamaglia, V., Sollazzo A. and Mattei, M. (2012). Fixed structure flight control design of an over-actuated aircraft in the presence of actuators with different dynamic performance, Proceedings of the 7th IFAC Symposium on Robust Control Design, Aalborg, Denmark, (CD-ROM).

Seron, M.M., De Dona, J.A., and Olaru, S. (2013). Fault tolerant control allowing sensor healthy-to-faulty and faulty-to-healthy transitions, IEEE Transactions on Automatic Control 57(7): 1657-1669.

Staroswiecki, M. (2010). On reconfiguration-based fault tolerance, Proceedings of the 18th Mediterranean Conference on Control and Automation (MED), Marrakech, Marocco, pp. 1681-1691.

Stevens, B.R. and Lewis, F.L. (1992). Aircraft Control and Simulation, Wiley Interscience, New York, NY.

Steffen, T. (2005). Control Reconfiguration of Dynamical Systems, Lecture Notes in Control and Information Science, Vol. 320, Springer-Verlag, Berlin/Heidelberg.

Tan, W. and Packard, A. (2008). Stability region analysis using polynomial and composite polynomial Lyapunov functions and sum-of-squares programming, IEEE Transactions on Automatic Control 53(2): 565-571.

Zhang, Y. and Jiang, J. (2008). Bibliographical review on reconfigurable fault-tolerant control systems, Annual Reviews in Control 32(2): 229-252.

Wang, Y. and Boyd, S. (2010). Fast model predictive control using online optimization, IEEE Transactions on Control Systems Technology 18(2): 267-278.

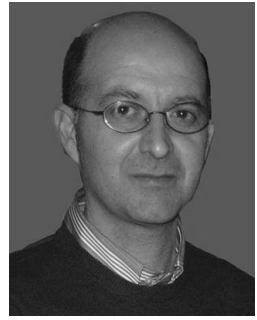

Giuseppe Franzè was born in 1968 in Italy. He received the $\mathrm{Ph} . \mathrm{D}$. degree in systems engineering from the University of Calabria in 1999. From 1994 to 2002 he was with the DEIS University of Calabria, Italy, first as an assistant researcher and since 2002 as an assistant professor. His current research interests include constrained predictive control, nonlinear systems, networked control systems, control under constraints, and control reconfiguration for fault tolerant systems.

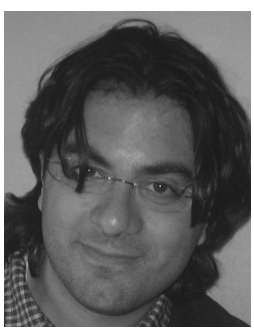

Angelo Furfaro was born in 1974 in Italy. He received the Ph.D. degree in systems and computer engineering from the University of Calabria, Italy, in 2004. He is an assistant professor at the University of Calabria, where he lectures on software engineering and embedded devices programming. His current research interests include modelling and simulation, modelchecking, and real-time systems.

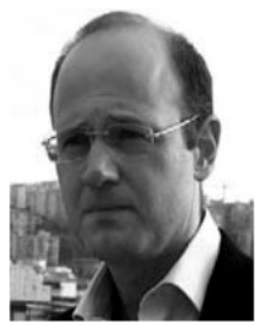

Massimiliano Mattei received the Laurea degree in aeronautical engineering and the Ph.D. in electronic engineering from the University of Napoli Federico II in 1993 and 1997, respectively. Professor Mattei is an expert in the area of modeling and control of plasmas in thermonuclear fusion reactors. He regularly collaborates with JET, ITER, Fusion for Energy, EFDA, ENEA, and many other worldwide institutions in this area. His research interests also include flight control and autonomous vehicles guidance, control and navigation. $\mathrm{He}$ is a professor of flight mechanics and control at the Second University of Napoli, where he is also the head of the Department of Industrial and Information Engineering.

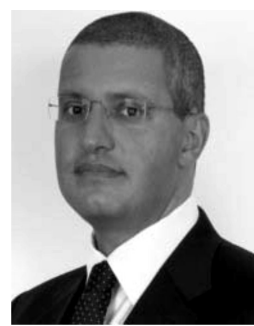

Valerio Scordamaglia was born in 1977 in Italy. He received the Ph.D. degree in electric and automation engineering from the University of Reggio Calabria, Italy, in 2008. Since 2009 he has been with the DIIES University of Reggio Calabria as an assistant professor. His current research interests include robust control, constrained predictive control, and control reconfiguration for fault tolerant systems.

Received: 29 January 2014 Revised: 11 June 2014 